\title{
IS THE WHEELCHAIR FENCING CLASSIFICATION FAIR ENOUGH? A KINEMATIC ANALYSIS AMONG WORLD-CLASS WHEELCHAIR FENCERS
}

\author{
Ying-ki Fung ${ }^{1}{ }^{1}$ Derwin King-Chung Chan ${ }^{2}, K_{i m}$ M. Caudwell ${ }^{2}$ Bik-chu Chow $^{3}$ \\ 1. Department of Rehabilitation Sciences, Hong Kong Polytechnic University, Hong Kong \\ 2. Dep. of Physical Education, School of Psychology and Speech Pathology, Curtin University, Perth, Australia \\ 3. Hong Kong Baptist University, Hong Kong
}

The purpose of this study was to employ a kinematic analysis to determine the extent to which the Wheelchair Fencing Classification (WFC) can reliably predict and classify wheelchair fencers' trunk functional ability, during WFC functional classification assessment condition (without supporting bar) and competition condition (with supporting bar). Participants were 14 world-class wheelchair fencers from Hong Kong, with 9 WFC category A and 5 WFC category B fencers. Participants performed wheelchair fencing actions (i.e., lunge and fast-return) in two conditions (i.e., standard WFC testing condition and wheelchair fencing in competition condition). The maximum trunk velocity and maximum trunk angle (i.e., range of movement) were motion-captured and analyzed by kinematic analysis. The results showed that WFC classification significantly correlated with the trunk functional ability in the WFC testing condition, but not in the competition condition. The functional ability indices were significantly higher in the competition condition than that in the WFC testing condition for fencers of both category A and B. The trunk functional ability of category A fencers was significantly higher than that of category B fencers in a WFC testing condition, but such patterns were not observed in the competition condition. We concluded that the WFC test might not be fair and reliable enough to classify fencers according to the impact of their impairments on wheelchair fencing competitive performance.

\section{Is the Wheelchair Fencing Classification Fair Enough?}

Use of kinematic analysis among world-class wheelchair fencers and in disability sport allows disabled individuals to participate in competition under fair and equitable condition (IWFC, 2008; Tweedy, 2002; Tweedy \& Vanlandewijck, 2011). Thus, it is important to have a functional classification system to ensure equitability (Tweedy \& Vanlandewijck, 2011; Wu \& Williams, 1999). In disability sports, the classification of functional ability takes into account the extent to which impairments affect sporting outcomes - this is conceptually different from disability classification in a clinical setting which focuses on physical symptoms (Tweedy \& Vanlandewijck, 2011). The use of functional classification systems allows disability athletes to be categorized into different groups according to their functional ability in respective sports (Tweedy \& Vanlandewijck, 2011). Indeed, due to the uniqueness and specific development of each disability sport event, no universal criterion can be defined for disability categorization across different sports; this raises issues of the reliability and validity of methods determining disability classification (Doyle, et al., 2004). Correspondingly, the current study employed a 
kinematic analysis to examine if the disability classification system of wheelchair fencing provided a good indication of players' functional ability in a competition setting.

\section{Disability Classification in Disability Sports}

Research consistently shows disability sports employ taxonomical methods of classification for equitable competition across athletes (Porretta \& Sherrill, 2005; Tweedy, 2002; Vanlandewijck \& Evaggelinou, 2003; Vanlandewijck \& Chappel, 1996; Williamson, 1997; Wu \& Williams, 1999). However, few attempts have been made to utilise evidence-based methods to compare the functional ability of players in different functional categories. Wu and Williams' (1999) study on the performance of swimmers in the 1996 Paralympic games found swimmers with varying levels of disability exhibited different levels of sporting performance, with swimmers of distinct types of impairment having similar chances of winning. The authors concluded that classification of swimmers according to their functional ability was reasonable. However, it is important to note that the study retrieved performance data (e.g., time and position) from the competition only, and it is possible that this data (i.e., sporting outcome) may not be entirely comparable to functional ability as could be assessed by a number of kinematic methods (e.g., range, speed, and power of motion). Recently, Beckman and Tweedy (2009) developed a test battery to evaluate the functional ability of disability runners (comprising a thirty metre sprint, standing broad jump, four bounds, a ten metre skip, running [in place], and split jumps). While the test battery exhibited good reliability and validity in predicting running performance, it was only tested among non-disabled individuals, meaning it may not be suitable for classifying the functional ability of disabled individuals (Beckman \& Tweedy, 2009). It remains unclear how well the existing classification systems reflect the functional ability of disability players in Paralympics summer sports (Chow, Chae, \& Crawford, 2000; Chow, Kuenster, \& Lim, 2003; Chow \& Mindock, 1999; Frossard, Smeathers, O'Riordan, \& Goodman, 2007).

\section{Wheelchair Fencing and Disability Classification.}

Wheelchair fencing is similar to able-bodied fencing, yet static, with fencers competing in wheelchairs fixed in place by metal frames and clamps to prevent tipping while maximising upper body movement (International Wheelchair and Amputee Sports Federation [IWAS], 2012). Players compete in foil, epée, and sabre (male only) events, with the aim of scoring 15 points against their target opponents in a three minute period, where a point is awarded for each strike on the opponents' target area (IWAS, 2012). Wheelchair fencers are classified into competitive categories through their scores on six World Fencing Classification (WFC) functional tests. The scores on these tests determine their competitive category (IWAS, 2011). As the attacking or defensive actions inherent in wheelchair fencing require trunk movement, the classification tests adopt a point-score system to assess trunk functional ability in terms of the range, strength, and speed of trunk movement and balance (IWAS, 2011). Points range from 0 to 3 , with 0 indicating no function and 3 points indicating normal execution (IWAS, 2011). Fencers with higher scores, and with greater trunk functional ability, are classified into category A, while others with lower trunk functional ability are classified into category B 
(category $\mathrm{C}$ is used for even lower trunk functional ability, however this category is combined with category B in IWC competitions). It is important to note that functional trunk classification relies on the subjective judgement of appointed classifiers, rather than objective and scientific assessment methods (e.g., motion analysis). In this case, the WFC result can potentially be influenced by the individual differences in classifier experience, sensory-bias, and the personal quantification of the scale anchors (e.g., "weak execution") among classifiers.

In order to determine how the impairments of trunk movement might affect wheelchair fencing performance, classification tests for wheelchair fencers should evaluate their trunk functional ability in a condition equivalent to a standard wheelchair fencing competition. During the WFC tests, fencers are required to sit on their own specially-made wheelchairs without holding the rims, spoke or any other support (IWAS, 2011). However, in competition, wheelchair fencers are allowed to make use of a supporting bar, fixed to the wheelchair (see Figure 1). Although the use of supporting bar is commonly applied to other disability sport events (e.g., wheelchair shot-put) for assisting players' balance (Chow, Chae, \& Crawford, 2000; Chow, Kuenster, \& Lim, 2003), it may also compensate the effect of lower functional ability (i.e., as ascertained by the WFC) on performance. For example, the WFC tests 2 and 4 require the athlete to move their centre of gravity to the point of losing balance; in a competitive scenario, this point may be extended by gripping the supporting bar.

Tweedy and Vanlandewijck (2011) have developed a statement of purpose for improving the validity of Paralympic classification: "...to promote participation in sport by people with disabilities by minimising the impact of eligible impairment types on the outcomes of competition" (p. 259). The authors advocate for research that incorporates objective and reliable measures of both impairment and functional ability (Tweedy \& Vanlandewijck, 2011). Methods used to classify the trunk functional ability of wheelchair fencers, which subsequently determine competitive classes, have been questioned in regard to their fairness and ecological validity in competitive wheelchair fencing settings (Fung, Chow, Fong \& Chan, 2010). The obtained WFC test score and functional ability classification may not equate to ability in wheelchair fencing competition. Kinematic analysis therefore offers an objective examination of body movement parameters (e.g., trunk functional ability), offering reliable information about the degree to which disability impairs performance in Paralympic competition.

\section{The Present Study}

The aim of this study was to preliminarily investigate if the trunk functional ability assessed in the WFC condition (without supporting bar) was comparable to that in the competition (IWFC) condition (with supporting bar). In order to examine the trunk functional ability in the two conditions, a number of functional indices associated with wheelchair fencing were used. We assessed wheelchair fencers' trunk functional ability by their trunk maximum velocity and trunk maximum angle (Czajkowski, 2005). Consequently, based on the fundamental principle of Paralympics sport classification (Tweedy \& Vanlandewijck, 2011), we hypothesized that the WFC classification (i.e., category A versus category B) would reliably 
predict the trunk function ability indices, and the prediction would be consistent across both WFC condition and competition condition. Specifically, we speculated that (a) the WFC condition would correlate positively with the trunk functional ability indices in the WFC condition as well as in the competition condition, (b) trunk functional ability indices in the competition condition (with supporting bar) would not significantly differ from that of the WFC condition (without supporting bar), and (c) category A fencers would perform better than category $\mathrm{B}$ fencers in both conditions.

\section{FIGURE 1}

A fencing wheelchair equipped with a supporting bar.

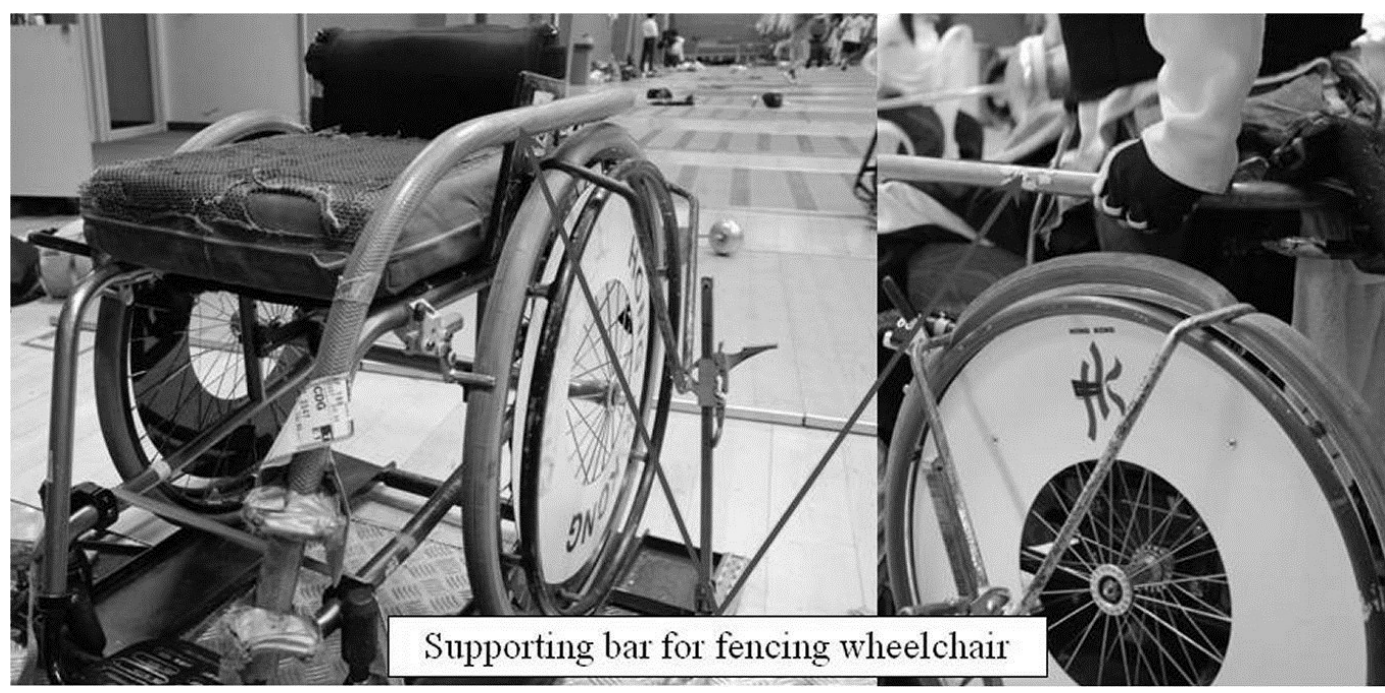

\section{Method}

\section{Participants}

After obtaining approval from the Research Ethics Committee of Hong Kong Baptist University, eight male and six female Hong Kong Team wheelchair fencers (mean age $=29.93, S D$ $=5.98)$ participated in the study. This sampling population comprised all squad members of the Hong Kong wheelchair fencing team in 2006. Competitors had at least 3 years' experience of competitive wheelchair fencing (mean $=6.57, S D$ $=3.76$ ), and had participated in a number of international competitions including the Paralympic games. Basedon participants' classification in previous international competitions, our sample consisted of nine WFC category A and five category B wheelchair fencers. Detailed demographic information of the participants is shown in Table 1. Participants provided informed consent about their participation rights (i.e., right to withdraw the study and data given at anytime) and confidentiality of the data before the experiment.

\section{Procedures}

After approximately fifteen minutes of warm-up, participants were instructed to perform a lunge (attack movement; see Figure 2a) and then a fast-return (defensive movement; see Figure $2 b$ ) with maximum speed in both the competition condition and WFC condition. The test under WFC condition was equivalent to the WFC test 4 (IWFC, 2008), and therefore 
participants were not permitted to hold the supporting bar (see Figure 2a and 2b). Whereas, the participants performed the same test again in the competition condition, with their non-fencing arms holding the supporting bar (see Figure $2 \mathrm{c}$ and d). To reduce carry over effects, the order of the tests taken under the two conditions was counter balanced. Participants performed five trials for each condition, and they were allowed to rest for as long as they deemed necessary after each trial.

\section{Experimental setup}

We followed the official rule of International Wheelchair Fencing Committee (IWFC, 2008) to setup the classification tests in both conditions. Firstly, an experimenter (or named classifier; IWFC, 2008) was presented opposite to the participants to simulate an environment similar to a wheelchair fencing competition, and more importantly to provide a target for the participants to perform a lunge. Secondly, the experimenter sat at the center of the seat, and was kept still in a standard defensive posture of wheelchair fencing during each trial. Thirdly, we adjusted the fencing distance between the participants and the experimenter to ensure participants had sufficient room to perform lunge and fast-return regardless of the individual differences in limb-length. During the distance adjustment, the experimenter and participant had to sit upright (rather than leaning forward or backward) at the centre of their wheelchairs, and the experimenter's upper limb was with shoulder in 90-degree abduction, and the 90-degree flexed elbow was parallel to participants' sagittal plane. The distance was adjusted until participants' fencing foils could reach the inner edge of the experimenter's forearm (see Figure 3).

\section{TABLE 1}

Demographic information

\begin{tabular}{|c|c|c|c|c|c|c|}
\hline Fencer Code & Gender & Cat. & Age & Years of Experience & Highest Level Competition & Diagnosis \\
\hline 1 & Male & A & 23 & 4 & WWFC & AP \\
\hline 2 & Male & $\mathrm{A}$ & 28 & 4 & WWFC & AP \\
\hline 3 & Male & A & 26 & 10 & PG & Para \\
\hline 4 & Male & A & 35 & 15 & PG & Polio \\
\hline 5 & Male & A & 32 & 3 & WWFC & ParaW \\
\hline 6 & Male & B & 36 & 9 & PG & ParaW \\
\hline 7 & Male & B & 36 & 9 & PG & ParaW \\
\hline 8 & Male & B & 40 & 5 & PG & ParaW \\
\hline 9 & Female & A & 22 & 5 & PG & AP \\
\hline 10 & Female & A & 28 & 5 & PG & Hemi \\
\hline 11 & Female & A & 35 & 12 & PG & ParaW \\
\hline 12 & Female & A & 23 & 3 & PG & Hemi \\
\hline 13 & Female & B & 23 & 4 & PG & ParaW \\
\hline 14 & Female & B & 32 & 4 & WWFC & ParaW \\
\hline
\end{tabular}

Note. Cat. $=$ category, WWFC $=$ World Wheelchair Fencing Championship, $\mathrm{PG}=$ paralympic games, $\mathrm{AP}=$ amputee, para $=$ paraplegia (walk with aid), $\mathrm{ParaW}=$ paraplegia (wheelchair-bounded), Hemi $=$ hemiplegia.

\section{FIGURE 2}

Trunk function ability tests in the two experimental conditions. 


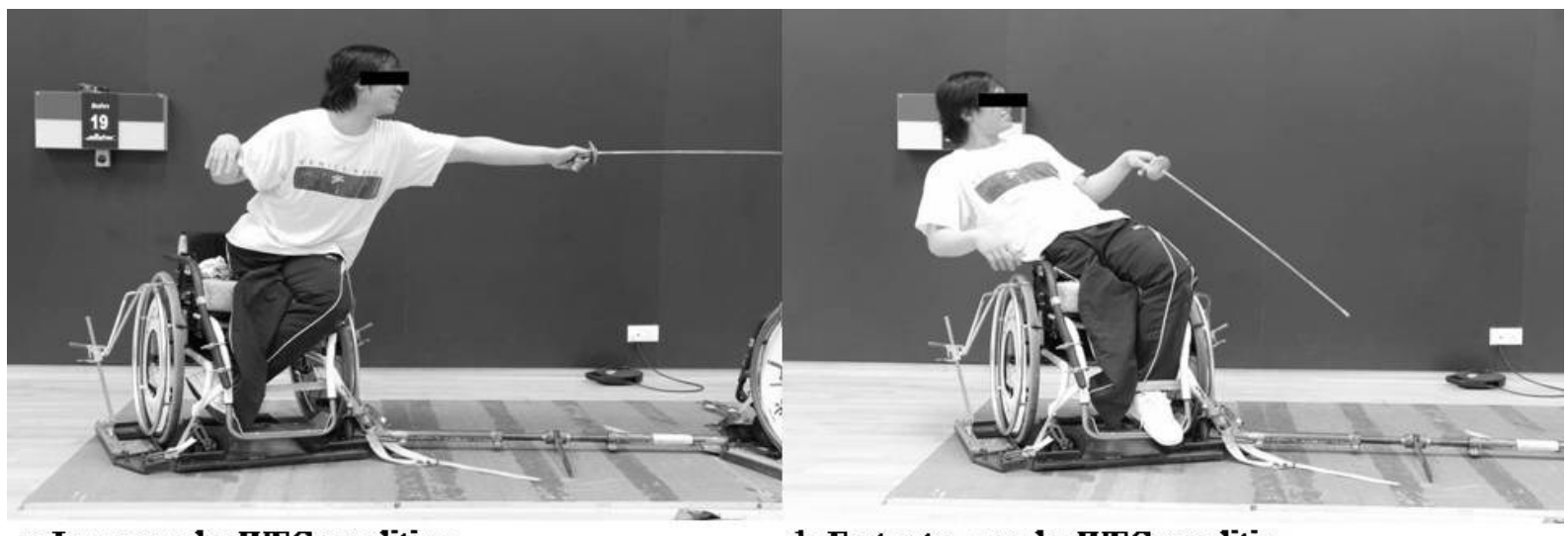

a Lunge under WFC condition

b. Fast-retum under WFC condition

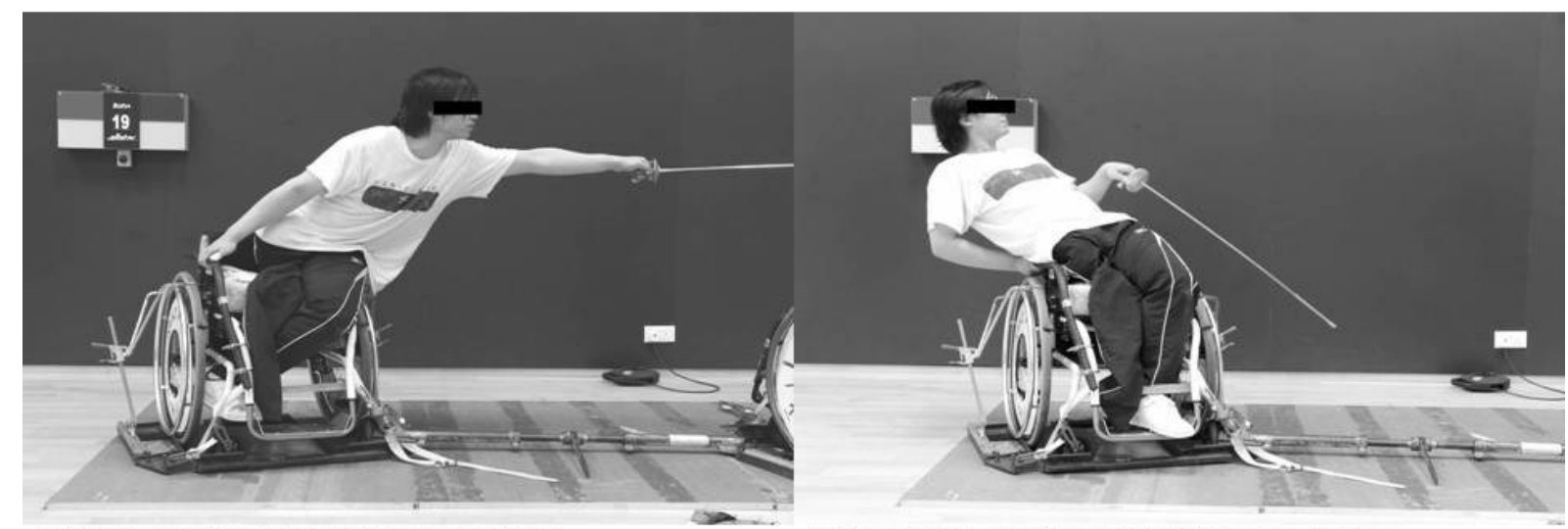

c. Lunge under competition condition

d. Fast-retum under competition condition

\section{FIGURE 3}

Distance normalization between experimenter and participant.

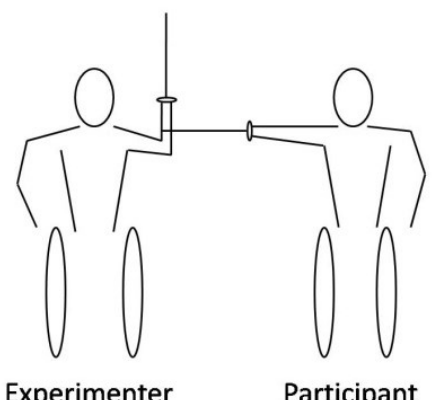

\section{Measures of trunk functional ability}

The trunk functional ability was assessed by 2 motion indices (i.e., maximum velocity and maximum angle) for both lunge and fast-return. The maximum velocity was the highest velocity of the sterna notch, whereas the maximum trunk angle was largest angle between two reference lines (see Figure 4): (a) the line between the shoulder of the fencing arm and the lilac crest of the fencing arm (line $\mathrm{SH}$ ), and (b) the vertical line perpendicular to the ground that went through the hip joint (line HX). Although the internal consistency of the assessments of maximum lunge velocity $\left(\alpha_{\mathrm{WFC}}=.94 ; \alpha_{\text {competition }}=.86\right)$, maximum lunge angle $\left(\alpha_{\mathrm{WFC}}=.89 ; \alpha_{\text {competition }}\right.$ $=.85)$, maximum fast-return velocity $\left(\alpha_{\mathrm{WFC}}=.94\right.$; 
$\left.\alpha_{\text {competition }}=.82\right)$, and maximum fast-return angle $\left(\alpha_{\mathrm{WFC}}=.96 ; \alpha_{\text {competition }}=.97\right)$ were excellent among the 5 trials, the scores from participants' best trial were used for data analysis.

\section{Motion capture}

A Sony 3CCD (DCR-TRV950E) digital video camera recorder was used to videotape motions $(50 \mathrm{~Hz})$ in a resolution of 300 mega-pixels per frame. The camera captured participants' motions (in the frontal plane) of each trial with a distance of 10 meters away from the wheelchair fencing frame, with the recording time adjusted to ensure all lunge or fast-return motions in each trial were completely videotaped (see Figure 5). Before filming, a 1 x 1 metre calibration board, located in the wheelchair fencing frame, was video-recorded for defining the global coordination volume. In addition, to enhance precision of motion capture, participants were advised to wear tight-fitted upper body clothing (or no clothing for male participants). The Peak Motus ${ }^{\circledR}$ Motion Measurement System (Peak Performance Technologies) was used to extract the motion formation from video, and then compute the maximum trunk velocity and angle of the lunge and fast-return movements. The extraction of motion data was then smoothed by Butterworth $2^{\text {nd }}$ order filter (Low-pass $=8 \mathrm{~Hz}$ ) before computation of the trunk functional indices.

\section{Data analysis}

To mitigate the effects of low sample size and non-normality, we adopted three non-parametric tests to analyze the data. Spearman's rank correlation coefficients (i.e., $\rho$ ) between the WFC classification and each trunk functional ability index were computed. The Wilcoxon
Signed-Rank test was employed to test difference of the trunk functional ability (the maximum angle and velocity on lunge and fast-return) between the classification and competition condition. The Mann-Whitney U-test was used to examine the difference of trunk functional ability between category A participants, and category B participants. We adopted a statistical significance level of $p<0.05$.

\section{Results}

Consistent with our hypotheses, we found a significant relationship between WFC classification and trunk functional indices in the WFC condition $(\rho=.54-.76 ; p<.01)$; however WFC classification was only related to maximum fast-return angle in the competition condition $(\rho=.69 ; p<.01)$. Inconsistent with our hypothesis, Wilcoxon Signed-Ranked tests showed that all trunk functional ability indices, including maximum lunge velocity and angle, and maximum fast-return velocity and angle, were higher when being assessed in the competition condition than in the WFC condition (all $p<.05$ ), with this pattern being observed across two disability categories (A and B). Nevertheless, Mann-Whitney U-tests revealed that the trunk functional ability of category A participants was higher than that of category B participants in the WFC condition only, in terms of maximum lunge angle, and maximum fast-return velocity and angle (all $p<.05$ ). However, the difference of maximum lunge velocity between the two categories was non-significant $(p>.05)$. In contrast to our predictions, no significant differences of trunk functional ability indices (i.e., maximum lunge velocity, maximum lunge angle, maximum fast-return velocity) were observed between the two categories when the tests were performed in 
the competition condition (all $p<.05$ ), apart from significant difference of the maximum fast-return angle $(p<.05)$. Table 2 displays these results in more detail, with Figure 6 providing a graphical illustration of the results.

\section{TABLE 2}

Descriptive statistic and tests results

\begin{tabular}{|c|c|c|c|c|c|c|c|c|c|}
\hline $\begin{array}{l}\text { Trunk } \\
\text { Functional } \\
\text { Ability }\end{array}$ & $\begin{array}{c}\text { Participants' } \\
\text { Categories }\end{array}$ & $\mathrm{N}$ & $\begin{array}{c}\text { Comp Score } \\
\text { Mean } \pm \text { SD } \\
\text { (Range) }\end{array}$ & $\begin{array}{c}\text { WFC Score } \\
\text { Mean } \pm \text { SD } \\
\text { (Range) }\end{array}$ & $\begin{array}{c}{ }^{\mathrm{a}} \text { Comp - Class } \\
\text { Mean Diff }\end{array}$ & \multicolumn{2}{|c|}{$\begin{array}{c}{ }^{\mathrm{b}} \text { Cat-A - Cat-B } \\
\text { Mean Diff }\end{array}$} & \multicolumn{2}{|c|}{$\begin{array}{c}{ }^{\mathrm{C}} \text { Cat-A \& Cat-B } \\
\text { Spearman } \rho\end{array}$} \\
\hline & & & & & & Comp & WFC & Comp & WFC \\
\hline \multirow{2}{*}{$\begin{array}{l}\text { Maximum } \\
\text { Lunge Velocity } \\
\left(\mathrm{ms}^{-1}\right)\end{array}$} & A & 9 & $\begin{array}{l}1.48 \pm 0.28 \\
(1.04-2.01)\end{array}$ & $\begin{array}{l}1.00 \pm 0.44 \\
(0.24-1.56)\end{array}$ & $0.47 * *$ & \multirow{2}{*}{0.08} & \multirow{2}{*}{0.60} & \multirow{2}{*}{0.15} & \multirow{2}{*}{$0.54 *$} \\
\hline & B & 5 & $\begin{array}{c}1.39 \pm 0.08 \\
(1.30-1.51)\end{array}$ & $\begin{array}{l}0.40 \pm 0.11 \\
(0.29-0.58)\end{array}$ & $0.99 *$ & & & & \\
\hline \multirow{2}{*}{$\begin{array}{l}\text { Maximum } \\
\text { Lunge Angle } \\
\text { (degree) }\end{array}$} & A & 9 & $\begin{array}{l}44.61 \pm 5.63 \\
(33.50-50.5)\end{array}$ & $\begin{array}{l}33.67 \pm 11.34 \\
(14.00-49.5)\end{array}$ & $10.94 * *$ & \multirow{2}{*}{4.81} & \multirow{2}{*}{$20.77 *$} & \multirow{2}{*}{0.43} & \multirow{2}{*}{$0.76^{* *}$} \\
\hline & B & 5 & $\begin{array}{l}39.90 \pm 5.87 \\
(33.00-46.5)\end{array}$ & $\begin{array}{l}13.10 \pm 3.31 \\
(8.00-16.5)\end{array}$ & $26.90 *$ & & & & \\
\hline \multirow{2}{*}{$\begin{array}{l}\text { Maximum } \\
\text { Fast-Return } \\
\text { Velocity }\left(\mathrm{ms}^{-1}\right)\end{array}$} & A & 9 & $\begin{array}{l}1.19 \pm 0.28 \\
(0.77-1.52)\end{array}$ & $\begin{array}{l}0.84 \pm 0.39 \\
(0.34-1.41)\end{array}$ & $0.36^{* *}$ & \multirow{2}{*}{0.09} & \multirow{2}{*}{$0.53 *$} & \multirow{2}{*}{0.17} & \multirow{2}{*}{$0.72 * *$} \\
\hline & B & 5 & $\begin{array}{l}1.01 \pm 0.22 \\
(0.91-1.43)\end{array}$ & $\begin{array}{l}0.31 \pm 0.17 \\
(0.13-0.59)\end{array}$ & $0.80 *$ & & & & \\
\hline \multirow{2}{*}{$\begin{array}{l}\text { Maximum } \\
\text { Fast-Return } \\
\text { Angle (degree) }\end{array}$} & A & 9 & $\begin{array}{c}64.67 \pm 21.41 \\
(42.00-108.00)\end{array}$ & $\begin{array}{c}58.39 \pm 24.32 \\
(29.00-104.00)\end{array}$ & $6.39 *$ & \multirow{2}{*}{$24.41 *$} & \multirow{2}{*}{$39.22 *$} & \multirow{2}{*}{$0.69^{* *}$} & \multirow{2}{*}{$0.76^{* *}$} \\
\hline & B & 5 & $\begin{array}{c}40.40 \pm 8.71 \\
(30.00-53.50)\end{array}$ & $\begin{array}{c}19.10 \pm 12.30 \\
(7.50-34.00)\end{array}$ & $21.20 *$ & & & & \\
\hline
\end{tabular}

Note . Comp $=$ competition condition; $\mathrm{WFC}=$ wheelchair fencing classification condition; Diff $=$ difference.

$* p \leq 0.05, * * p \leq 0.01$

${ }^{a}$ Wilcoxon Signed-Rank tests examined the difference between two categories of fencers.

${ }^{b}$ Mann-Whitney $\mathrm{U}$ testswere employed to test the difference between two categories of fencers.

'Spearman's ranked correlation revealed the associations between the WFC classification and trunk functional indices.

\section{FIGURE 4}

Computation of maximum trunk angle. SH represents the line between the shoulder of the fencing arm and the lilac crest of the fencing arm, and HX represents the vertical line perpendicular to the ground that went through the hip joint.

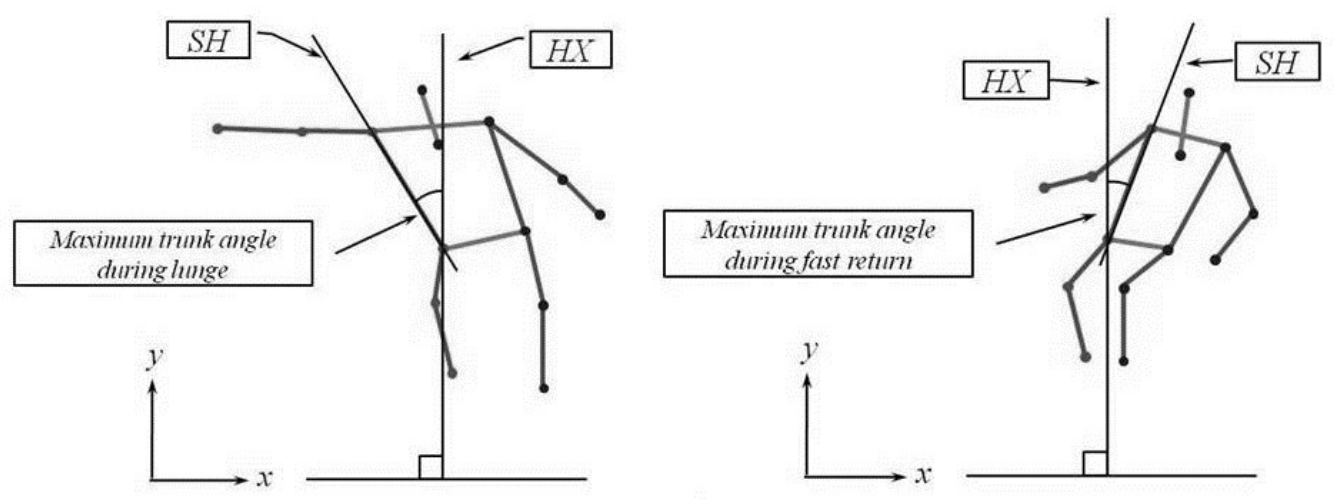




\section{FIGURE 5}

Experimental setup for right and left fencing arm.

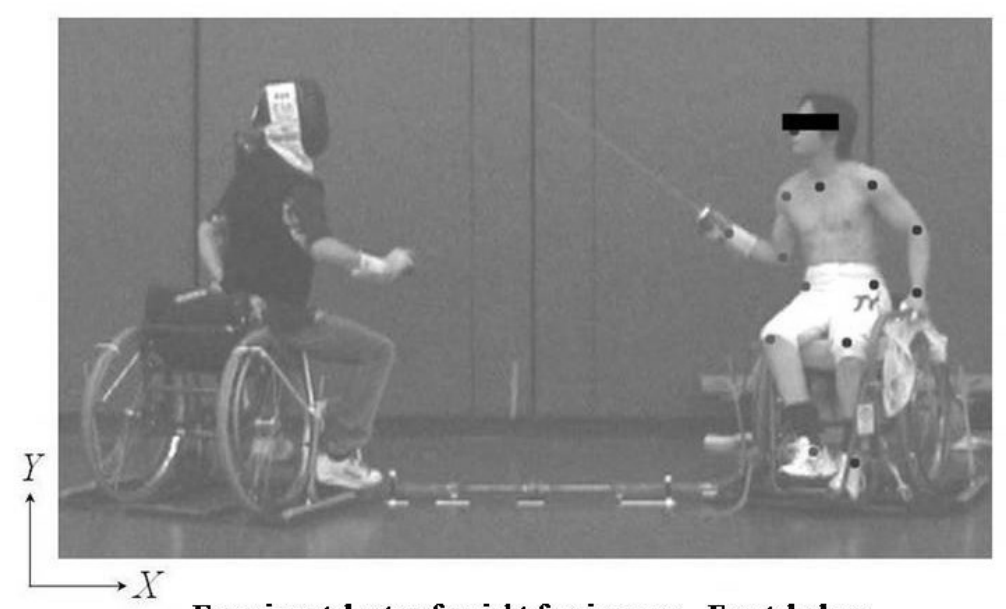

Experimental setup for right fencing arm - Frontal plane

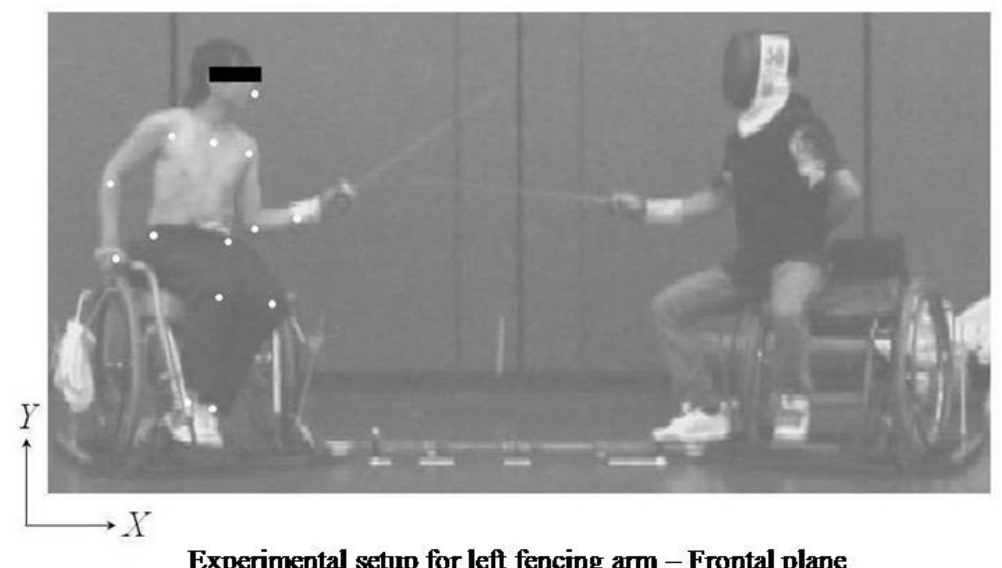

Experimental setup for left fencing arm - Frontal plane

\section{FIGURE 6}

Functional ability between Category A and B participants with and without using the supporting bar. The functional ability in the $\mathrm{y}$-axis is the mean standardized score of all trunk functional ability indices. $* p<0.05$.

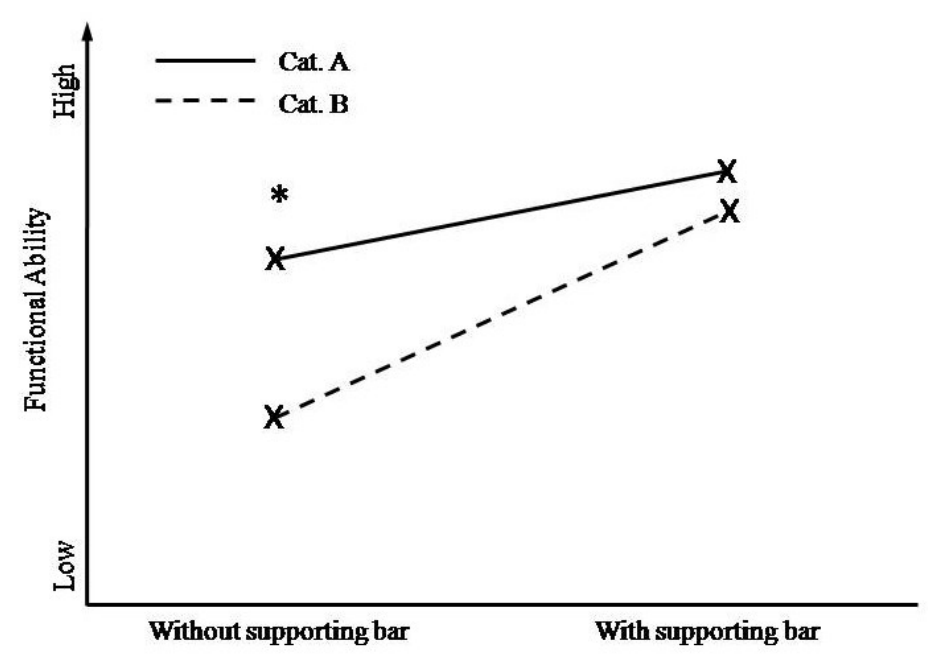




\section{Discussion}

In the present study, we attempted to employ a kinematic analysis to test the reliability and ecological validity of the Wheelchair Fencing Classification (WFC) regarding two wheelchair fencing actions: lunge and fast-return. We hypothesized that the WFC tests which measure trunk functional ability would provide a reliable assessment on the effect of impairment on wheelchair fencing functional performance indices, and ascertain a fair categorization among disability fencers according to their trunk functional ability. Our overall findings did not fully support these hypotheses. Although WFC classification was found to reliably predict trunk functional ability (assessed in the WFC condition), the results showed that the existing WFC test (4) tended to underestimate fencers' trunk functional ability in competition settings. Further, the two disability categorizations (A and B) failed to differentiate players' wheelchair fencing performance (IWFC competition condition) regarding most motion parameters.

\section{Ecological Validity of the WFC tests}

The central premise of classification in disability sport is to identify athletes' functional ability by a series of specified performance determinants, meaning the results of classification should consistently predict sport performance or the functional potential of competitors (Tweedy \& Vanlandewijck, 2011; Wu \& Williams, 1999). The WFC classification was only associated with the trunk functional ability assessed in the standard WFC condition (without supporting bar), but not with most of the indices used in the competition condition (without supporting bar; IWFC, 2008). Moreover, we found that fencers obtained better trunk functional indices with the aid of supporting bar, suggesting the setup of the WFC test might undermine fencers' actual trunk functional in a competition setting.

It is interesting to discuss why the WFC test underestimated fencers' trunk functional ability more among category $B$ fencers than it did among category A fencers. According to the WFC (IWFC, 2008), category B fencers should have poorer sitting balance than category $\mathrm{A}$, and such functional impairment should affect their wheelchair fencing performance permanently to some extent, regardless of training. However, it appeared that category B fencers attained trunk functional ability levels comparable to that of category A fencers in a competition condition, and the effect of supporting bar was apparently more advantageous on category $B$ fencers than category A fencers. This result raises issues regarding the degree to which the use of supporting aids (e.g., supporting bar) in disability sports may compromise the effect of impairment on functional performance, and suggests the classification system should be adapted accordingly, to ensure fair and equitable competitions.

In recent years, the use of supporting equipment, strapping techniques, or other assistive aids are becoming popular and are increasingly recognized in disability sports. We suggest that such classification systems should take these and other supporting aids into account when assessing the impact of athletes' impairment on sport performance (Burkett, 2010; Tweedy \& Vanlandewijck, 2011). Some disability sports have already attempted to do so in their classification systems. For instance, during the classification of wheelchair basketball, players are asked to use the same wheelchair and 
strapping method as they do during the competition, incorporating the effect of the equipment on sport performance in classification (IWBF, 2010). Future studies should examine if the supporting bar in the competition setting of wheelchair fencing may somewhat compromise the negative effects of functional impairment on wheelchair fencing performance, and how the WFC tests could account for such comprising effects in classifying competitors.

\section{Reliability of the WFC Tests}

While the International Paralympics Committee is committed to improving the selective classification systems for Paralympic sports (Tweedy \&Vanlandewijck, 2011), classification tests should allow assessment of performance-related functional criteria with a sufficient level of reliability and precision, and clear standard of classification (also see IWFC, 2008). Our results did not fully support these promises for the existing classification test of wheelchair fencing. Although the WFC classification showed statistically significant relationships with all trunk parameters in the WFC condition, it failed to predict most trunk functional indices in the competition condition, and the magnitude of correlations between classification and performance indices were far lower than that seen in a disability swimming context (Wu \& Williams, 1999). Indeed, category A fencers should intuitively display better trunk functional ability than category B fencers, which was not the case in terms of the maximum lunge velocity in the WFC condition and most trunk functional indices in the competition condition.

The lack of significant differences could result from a number of factors, including low statistical power and small effect sizes of WFC classification. Nevertheless, the possibility of miscategorization in WFC classification should not be ignored as it may increase the extent of measurement error in the significant tests. This indeed addresses an important issue of classification in disability sport, that functional ability based on the observation of classifiers alone might not be adequate, comprehensive, and sufficiently reliable. Future research is warranted to clearly define disability categories based on scientific evidence, and develop more advanced assessment methods to improve the reliability and precision of functional ability assessment (Beckman \& Tweedy, 2009; Burkett, 2010; Tweedy \& Vanlandewijck, 2011).

\section{Limitations and Future Directions}

A number of limitations in this study should be noted. First, classification in disability sport should reflect the permanent nature of functional impairments, but the cross-sectional design of the study did not permit us to examine this assumption, thus it is important to employ longitudinal studies to provide evidence regarding test-retest reliability of classification. Second, although the current sample comprised the whole elite wheelchair fencing population in Hong Kong, the size of this sample was indeed quite small; it might be useful to recruit larger sample to enhance the statistical power of the analyses. Third, our sample was only limited to world-class wheelchair fencers, so our findings might be affected by a ceiling effect. It may be worthwhile for further research to increase the coverage of the sample, and ascertain if our findings could be generalized to disability athletes of different levels, and from different sport events. Finally, the two functional criteria 
of wheelchair fencing (i.e., maximum trunk angle and velocity) as assessed in the present study might not be adequate to provide a comprehensive evaluation of all performance-related functional ability. Therefore, the reliability and validity of classification tests regarding aspects such as players' limb deficiency, hypertonia, ataxia, athetosis, and short stature remained unexplored.

\section{Conclusion}

The current study presented a preliminary kinematic analysis to examine the trunk functional ability of category A and B fencers under the IWAS wheelchair fencing classification. The results indeed did not fully support the ecological validity and reliability of the existing classification system. In conclusion, the existing classification for wheelchair fencing should be refined, particularly for the adaptation of the use of supporting equipment in competition setting. The introduction of motion analysis might be a future solution to enhance the accuracy and reliability of disability classification in sport.

\section{References}

Beckman, E. M., \& Tweedy, S. M. (2009). Towards evidence-based classification in Paralympic athletics: evaluating the validity of activity limitation tests for use in classification of Paralympic running events. British Journal of Sports Medicine, 43(13), 1067-1072.

Burkett, B. (2010). Technology in Paralympic sport: performance enhancement or essential forperformance? British Journal of Sports Medicine, 44(3), 215-220.

Chow, J. W., Chae, W., \& Crawford, M. J. (2000). Kinematic analysis of shot-putting performed by wheelchair athletes of different medical classes. Journal of Sports Sciences, 18(5), 321-330.

Chow, J. W., Kuenster, A. F., \& Lim, Y. T. (2003). Kinematic analysis of javelin throw performed by wheelchair athletes of different functional classes. Journal of Sports Science and Medicine, 2(2), 36-46.

Chow, J. W. \& Mindock, L. A. (1999). Discus throwing performances and medical classification of wheelchair athletes. Medicine \& Science in Sports \& Exercise, 31(9), 1272-1279.

Czajkowski, Z. (2005). Understanding fencing: The unity of theory and practice. New York: SKA Swordplay Books.

Doyle, T. L. A., Davis, R. W., Humphries, B., Dugan, E. L., Horn, B. G., Shim, J. K., \& Newton, R. U. (2004). Further evidence to change the medical classification system of thenational wheelchair basketball. Adapted Physical Activity Quarterly, 21(1), 63-70.

Frossard, L., Smeathers, J., O'Riordan, A., \& Goodman, S. (2007). Shot trajectory parameters in gold medal stationary shot-putters during world-class competition. Adapted Physical Activity Quarterly, 24(4), 317-331.

Fung, Y. K., Chow, B. C., Fong, D. T. P., \& Chan, K. M. (2010, July). A kinematic analysis of trunk ability in wheelchair fencing: a pilot study. Paper presented at the XXVIII International Conference on Biomechanics in Sports, Marquette, USA.

International Wheelchair Basketball Federation (2010). Official player classification manual.IWBF Player Classification Commission.

International Wheelchair \& Amputee Sports 
Federation. (2011). IWF rules for competition: Book 4 - Classification rules.

International Wheelchair \& Amputee Sports Federation. (2012). Wheelchair Fencing: Rules. Retrieved from http://iwasf.com/iwasf/index.cfm/sports/iwa s-wheelchair-fencing/rules/

International Wheelchair Fencing Committee (2008). Classification. International wheelchair fencing rules for competitions (Vol. 4). Retrieved from the website of International Wheelchair Fencing Committee:http://www.iwfencing.com/archi ves/rules/WF\%20Classification\%20Rules.p $\mathrm{df}$

Porretta, D. L., \& Sherrill, C. (2005). APAQ at Twenty: A documentary analysis. Adapted Physical Activity Quarterly, 22(2), 119-135. Tweedy, S. M. (2002). Taxonomic theory and the ICF: Foundations for a unified disability athletics classification. Adapted Physical Activity Quarterly, 19(2), 220-237.

Tweedy, S. M., \& Vanlandewijck, Y. C. (2011). International Paralympic Committee position stand - Background and scientific rationale for Classification in Paralympic Sport. British Journal of Sports Medicine, 45(4), 259-269.

Vanlandewijck, Y. C., \& Chappel, R. J. (1996). Integration and classification issues in competitive sports for athletes with disabilities. Sports Science Review, 5, 65-88.

Vanlandewijck, Y. C., \& Evaggelinou, C. (2003).Porportionality in wheelchair basketball classification.Adapted Physical Activity Quarterly, 20, 369-380. participants with disabilities: A proposal. Palaestra, 13(2), 44-48.

Wu, S. K., \& Williams, T. (1999). Paralympic swimming performance, impairment, and the functional classification system. Adapted Physical Activity Quarterly, 16(3), 251-270. 